Instituto Internacional de Investigación y Desarrollo Tecnológico Educativo INDTEC, C.A.

DOI: https://doi.org/10.29394/Scientific.issn.2542-2987.2019.4.11.12.239-259

OAI-PMH: http://www.indteca.com/ojs/index.php/Revista Scientific/oai

Artículo Original / Original Article

\title{
La Gerencia de las Direcciones de Cultura y la Responsabilidad Social Universitaria desde una Cosmovisión Integrativa
}

\author{
Autora: Sandra Isabel García Arévalo \\ Universidad Centroccidental "Lisandro Alvarado", UCLA \\ sandragarciarte@gmail.com \\ Lara, Venezuela \\ https://orcid.org/0000-0001-6338-8960
}

Resumen

La universidad, como organización responsable, mediante su función extensionista produce impactos, posee una Responsabilidad Social directamente relacionada con la misión universitaria y sus procesos de gestión. El propósito de este estudio es generar un constructo teórico sobre la gerencia de las Direcciones de Cultura y la Responsabilidad Social Universitaria desde una cosmovisión integrativa. El proceso investigativo se cimentó en un enfoque cualitativo, postura paradigmática emergente aclarativa, sustentada en el método fenomenológico-hermenéutico. Se utilizó la técnica de la entrevista a profundidad, el tratamiento de la información se realizó mediante la categorización, codificación y triangulación, permitiendo la interpretación del fenómeno investigado y generar un constructo teórico desde los fundamentos epistemológicos, ontológicos, axiológicos, filosóficos, praxeológicos y teleológicos relacionados con la gerencia de las Direcciones de Cultura y la responsabilidad social, a partir de las experiencias de cada informante clave, desde una cosmovisión integrativa. La extensión cultural permite formar a estudiantes y comunidades en conocimientos, saberes y experiencias, en el ámbito universitario, desde un aprendizaje multidisciplinario. Se concluye que las universidades deben proporcionar espacios de intercambio entre conocimientos y saberes con una postura crítica y creativa para la formación integral e interdisciplinaria dirigida al bienestar físico y mental, fortificando la formación profesional, el accionar, científico, cultural, tecnológico y ambiental hacia el beneficio de la colectividad.

Palabras clave: cultura; responsabilidad; universidad.

Fecha de Recepción: 19-06-2018

Fecha de Aceptación: 27-08-2018 


\title{
The Management of Directorate of Culture and University Social Responsibility from one Integrative Cosmovision
}

\begin{abstract}
The university, as a responsible organization, through its extension function produces impacts, possesses a Social Responsibility directly related to the university mission and its management processes. The purpose of this study is to generate a theoretical construct on the management of the Directorates of Culture and University Social Responsibility from an integrative worldview. The investigative process was based on the qualitative approach, with an emergent paradigmatic position clarifying, based on the phenomenological-hermeneutical method. The technique of in-depth interview was used, the information was processed through categorization, coding and triangulation, allowing the interpretation of the phenomenon investigated and generating a theoretical construct from the epistemological, ontological, axiological, philosophical, praxeological and teleological foundations related to the management of the Culture Directorates and social responsibility, based on the experiences of each key informant, from an integrative worldview. The cultural extension allows to form students and communities in knowledge, knowledge and experiences, in the university environment, from a multidisciplinary learning. It is concluded that universities should provide spaces for exchange between knowledge and knowledge with a critical and creative stance for comprehensive and interdisciplinary training aimed at physical and mental well-being, strengthening professional training, action, scientific, cultural, technological and environmental towards the benefit of the community.
\end{abstract}

Keywords: culture; responsibility; university.

Date Received: 19-06-2018

Date Acceptance: 27-08-2018 


\section{Introducción}

El inicio del tercer milenio ha representado para el mundo profundas transformaciones algunas de ellas reflejadas en el modelo educativo vigente en Venezuela desde 1999, fundamentado en la filosofía de la democrática en la direccionalidad que requiere redimensionar las actividades económicas, sociales empresariales, y educativas, con el fin de propiciar la participación de las comunidades en proyectos que se orienten a la atención a sus grupos internos y externos, entre ellos las organizaciones empresariales.

El concepto de Responsabilidad Social Empresarial, paulatinamente ha migrado tanto en su expresión filosófica, como en su expresión práctica, hacia otros tipos de organizaciones, entre las cuales están las universidades en su función extensión instrumento de primer orden como organizaciones socialmente responsables, por tanto es fundamental proyectar una nueva imagen gerencial eficiente y eficaz en el sector universitario y para lograrlo es fundamental optimizar la acción de la gerencia extensionista, espacio funcional donde se inscriben las Direcciones de Cultura, atinente a lo descrito en la Ley de Universidades (1970), artículo 138:

En cada Universidad, adscrita al Rectorado, funcionará una Dirección de Cultura, la cual fomentará y dirigirá las actividades de extensión cultural de la Universidad, contribuyendo a la formación del alumnado y a la difusión de la ciencia y la cultura en el seno de la colectividad (pág. 18).

De acuerdo a éste artículo, las Direcciones de Cultura tienen como primordial función efectuar actividades de extensión cultural en las universidades, también refiere la proyección científico-cultural, por otra parte, independientemente de que su denominación en las Instituciones de Educación Universitaria (IEU) puede variar, porque no todas las universidades han aplicado esta disposición legal, aunque tienen una instancia que se encarga la actividad cultural, para efectos de este trabajo se utilizará la 
denominación Direcciones de Cultura.

La tarea principal de las Direcciones de Cultura a través de la extensión cultural, como lo refiere la ley, una labor integrativa en la vinculación universidad-sociedad, por medio de las expresiones artísticas y culturales, basadas en la inclusión social en correspondencia con los principios y valores institucionales en beneficio de la humanidad. Como se observa, la gerencia cultural que desarrollan las Direcciones de Cultura universitaria en Venezuela, además de estar relacionada directamente con la normativa legal nacional, se enmarca en la filosofía de gestión de las instituciones universitarias y el accionar propio de cada casa de estudios superiores, encargada de desarrollar programas y proyectos del sector referido.

En la misma idea, Santos (2005), citado en Sarmiento (2007): presenta un concepto de gerencia cultural donde visualiza las dependencias de cultura universitaria como sistemas: "Un conjunto de elementos integrados que busca hacer realidad anhelos y satisfacer necesidades de la comunidad, donde todas las áreas de su organización son importantes" (pág. 38). El contexto cultural debe engendrar un trabajo sinérgico integrativo como soporte a las necesidades de las comunidades aunado a los fines institucionales.

En este marco de referencia se pretende la construcción de la gerencia cultural como una red integrada donde todas las áreas son importantes y requieren directores de cultura que actúen como eje articulador al cambio organizacional en las instancias universitarias. Por cuanto, las Direcciones de Cultura deben responder eficazmente a las demandas y necesidades del colectivo en correspondencia con la inversión social en programas y proyectos, donde se establezca la relación efectiva costo-beneficio social.

Asimismo, el trabajo realizado por la gerencia de las Direcciones de Cultura mediante la extensión cultural se encuentra dentro de la misión educativa universitaria como lo afirma Pérez (2016): "La universidad venezolana tiene que abordar de manera perentoria el proceso de integralidad 
de sus funciones universitarias" (pág. 64); conceptúa las funciones universitarias como parte exhaustiva de la labor instructiva del alma mater apoyándose, interrelacionándose y enriqueciéndose recíprocamente, por ello considera la extensión como una función integradora de saberes universitarios donde las Direcciones de Cultura tienen la misión de multidireccionar las relaciones universidad-comunidad hacia la transformación socio-cultural del país.

La gerencia cultural en el ámbito universitario constituye una transversalidad extensionista, al actuar como una red integradora de funciones en el contexto de la planificación estratégica, desarrollo de proyectos y programaciones permanentes que permiten abordar realidades de forma exhaustiva, donde se incluye la interrelación de diferentes disciplinas profesionales y artísticas que inducen a redefinir el mundo y su crisis actual, por ello las universidades tienen un prestigio ganado gracias a la formación profesional y el abordaje de la problemática social en Venezuela.

El laberinto debe afrontarse desde una perspectiva transdiciplinaria con esquemas cognitivos que puedan crear lazos entre las disciplinas, al respecto Morín (2002): indica que existe una “...inadecuación cada vez más amplia entre nuestros saberes desunidos, divididos, compartimentados y por el otro, realidades o problemas cada vez más poli disciplinarios, transversales, multidimensionales..." (pág. 14). Los conocimientos y los saberes deben visualizarse como totalidad con la extensión cultural, se activa el pensamiento crítico, la creatividad, la conexión científico cultural, la ciencia maneja con facilidad los hechos y el arte los significados, se trata de intercambiar estas vivencias para gestar nuevas propuestas, desde la extensión cultural.

Las Direcciones de Cultura universitaria, forman parte de la conexión con los grupos internos y externos de las Instituciones de Educación Universitaria, sin embargo, no han sido visionadas institucionalmente, como actoras relacionadas directamente con el cumplimiento de la (RSU) en visión 
de Chirinos y Pérez (2016), define: “...debe incorporarse en todas las universidades para la creación y difusión del conocimiento científico en función de garantizar aporte de competencias, cualidades y capacidades en las comunidades, lo que trae como respuesta el desarrollo sostenible..." (pág. 105); esta enunciación, resalta como la (RSU) debe fortalecer la vinculación universidad-entorno social bajo el enfoque gerencia humanista hacia la integración de conocimientos científicos y culturales.

Por tanto, las Direcciones de Cultura forman parte de la transversalidad con la difusión y extensión cultural que se moviliza como un fluido transformador del conocimiento, en el contexto de la planificación estratégica, desarrollo de proyectos y programaciones permanentes que permiten abordar realidades de forma sistémica donde se incluye la interrelación de diferentes disciplinas. Del mismo modo, Jaspe (2010), refiere: “...Confusión o desconocimiento de fines, diversidad conceptual... baja influencia de la universidad ante problemas sociales... subutilización de recursos y capital humano, ínfima asignación presupuestaria, falta de sistematicidad" (pág. 106).

Asimismo, las diversas visiones en cuanto a los objetivos de la extensión cultural dificultan el logro de metas, disipa esfuerzos de recursos humanos, aunado asignaciones presupuestarias descontextualizadas respecto a las necesidades culturales impide el desarrollo de proyectos interdisciplinarios dirigidos a la formación de estudiantes, profesores, comunidades y público en general, el trabajo continuo sin mecanismos de evaluación apropiados, todas estas condicionantes inciden en el bajo logro de los objetivos y permiten apreciar la problemática que enfrenta la gerencia en las Direcciones de Cultura Universitaria y su cometido entrelazado con el desarrollo de la metamorfosis sociocultural.

A su vez, desde el acercamiento dialógico con algunos gestores de la gerencia cultural universitaria que fungieron como informantes preliminares, tomados como referencia para indagar sobre su percepción acerca de la 
gerencia de las Direcciones de Cultura con relación a la Responsabilidad Social Universitaria, en este sentido, el Informante Preliminar 1 (IP1), opina que: La responsabilidad social de las universidades contempla la formación académica es decir profesionales, divulgación de investigaciones, la solución de problemas sociales en: salud, ingeniería, finanzas, aunque puede ser más amplia, las Direcciones de Cultura pueden participar en la formación integral de los estudiantes para que aprendan y aprehendan otros espacios cognitivos fuera su área disciplinar (IP1).

Por otra parte, el Informante Preliminar 2 (IP2), afirma que: Las funciones de las Direcciones de Cultura trascienden a las actividades de divulgación y promoción del hecho estético, se debe mejorar y unificar su nivel y ubicación en la estructura organizacional para otorgarle el apoyo administrativo y la acreditación curricular a la extensión para beneficio de las comunidades que reclaman profesionales con una visión transdisciplinaria en la solución de sus problemas sociales (IP2).

Lo anteriormente expuesto por los actores culturales, resalta las debilidades de la problemática referida por algunos autores sobre la gerencia cultural universitaria donde existe diversidad de criterios en cuanto a la ubicación en la estructura organizacional, desvinculación de las funciones universitarias, la falta de acreditación curricular para la extensión, contradicción en la normativa legal respecto a sus funciones científicoculturales, subutilización de recursos humanos, restricciones presupuestarias que afectan la formación sistémica de los estudiantes y la vinculación universidad-sociedad.

En este contexto, esta investigación, procura visualizar la manera cómo las Direcciones de Cultura del alma mater, el accionar gerencial, su relación e interconexión con el cumplimiento de la Responsabilidad Social Universitaria (RSU), desde una cosmovisión integrativa. Con el propósito de sistematizar el recorrido epistémico de la investigación surgieron las siguientes interrogantes: 
¿Cuáles son las percepciones que poseen los agentes gerenciales de las Direcciones de Cultura universitaria?,¿Cómo interpretar las percepciones gerenciales acerca del accionar gerencial de las Direcciones de Cultura universitaria?,¿Cómo se integran los postulados filosóficos, axiológicos y praxeológicos de la (RSU) con el accionar gerencial de las Direcciones de Cultura universitaria?,¿Cómo configurar un constructo teórico acerca de la gerencia de las Direcciones de Cultura y los basamentos epistemológicos de la Responsabilidad Social Universitaria desde una cosmovisión integrativa?.

Por tanto esta investigación pretende visualizar desde una cosmovisión integrativa la conexión, relación e Interarticulación del accionar gerencial de las Direcciones de Cultura de las universidades venezolanas, con los postulados filosóficos, axiológicos y praxeológicos de la (RSU), para lo cual será necesario develar e interpretar las percepciones que poseen los agentes gerenciales de las Direcciones de Cultura universitaria, imbricar los postulados filosóficos, axiológicos y praxeológicos, con el accionar gerencial de las Direcciones de Cultura universitaria y configurar un constructo teórico sobre la gerencia de las Direcciones de Cultura y la Responsabilidad Social Universitaria desde una cosmovisión integrativa.

\section{Análisis de los planteamientos teóricos}

\subsection{Gerencia Universitaria}

En el nuevo milenio la gerencia de las organizaciones posee retos en el desempeño de sus funciones, en un entorno dinámico y heterogéneo, al respecto, González (2015), señala: “El desafío de los gerentes y empresarios es propiciar un estilo gerencial donde se estimule el compromiso y participación de los trabajadores..." (pág. 105). Los gerentes deben respaldar un estilo gerencial que estimule y fortalezca el compromiso e identidad de los colaboradores, defina las condiciones para la construcción y solidez de un clima y una cultura organizacional que estimule a todos a ser autores del éxito 
organizacional.

Los líderes gerenciales deben motivar a sus seguidores mediante el compromiso y la participación en la búsqueda de un buen clima organizacional que permita el desempeño de los participantes como equipos de alto nivel movidos por la sinergia hacia el logro de las metas desde un concepto de excelencia y calidad. La gerencia universitaria tiene el compromiso de formar el talento humano requerido en todos los ámbitos disciplinares, egresando profesionales calificados y cualificados en este propósito, Rivero (2011): indica que los gerentes universitarios deben propiciar "Un sistema de trabajo en un ambiente laboral humano donde se cultive el aprecio, la confianza, el respeto, la motivación, el respeto por la vida en el planeta y prevalezca el poder de las ideas..." (pág. 30). La gerencia universitaria debe fomentar la motivación, respeto, el altruismo, responsabilidad, fraternidad, aprecio, tolerancia, trabajo en equipo en una red integrada de saberes y conocimientos, el aprendizaje en redes, enmarcados en el pensamiento crítico, la cultura, la innovación y la sostenibilidad, con la corrección de los impactos y la prevención de aquellos ocasionados por las actividades desarrolladas en las universidades, situación que puede ser asimilada por los estudiantes, docentes, grupos internos y externos para implementar estos modelos de gestión en escenarios propios de las áreas profesionales.

En este sentido, Goyo, Figueredo, Méndez, Chirinos y Rivero (2012): "enfatizan que el rol socioeducativo de la universidad se desarrolla mediante procesos consustancialmente articulados entre sí, relacionados con la dinámica social; con procesos gerenciales, que respondan a teleologías distintas, pero dialécticamente Interpenetradas" (pág. 112). Las Direcciones de Cultura universitaria, deben desarrollar un trabajo permanente a través de la divulgación ciencia y la cultura, el trabajo de estas dependencias, se delimita en actividades de extensión cultural en diversas modalidades, la divulgación científica es difusa, independientemente de su carácter normativo. En el 
mismo contexto, Tünnermann (2013), visualiza la universidad del futuro como: “...una Universidad donde las ciencias, las humanidades y las artes encuentren un alero propicio; la innovación, la imaginación y la creatividad su morada natural" (pág. 14).

La gerencia cultural universitaria es un campo altamente interdisciplinario con prácticas desde la integración de los saberes se puede coordinar eficaz y eficientemente un sistema de intervención cultural en la búsqueda del conocimiento transformador a través de la gestión de extensión cultural universitaria, dirigida a optimizar la preparación del recurso humano.

\subsection{Responsabilidad Social Universitaria}

En el contexto mundial globalizado, competitivo y dinámico, las organizaciones centradas en ampliar sus horizontes tienen un nuevo reto y por consiguiente en el sector universitario como formador de los futuros profesionales involucrados con el devenir gerencial para desarrollo del país, es pertinente considerar y aprehender el concepto de Responsabilidad Social Universitaria tanto en sus aspectos filosóficos como praxeológicos.

Al respecto, López, Zalthen, Hernández y Cervantes (2016), afirman que: "La RSU es una política de gestión de calidad ética y moral que busca formar personas con valores y compromisos sociales mediante el logro de las políticas institucionales establecidas como comunidad universitaria" (pág. 307). La investigadora se identifica con el criterio de estos autores y afirma que la Responsabilidad Social Universitaria está directamente relacionada con la misión universitaria, bajo un adeudo social activo, democratizador orientado a la solución de problemas y la transformación sociocultural del individuo.

En esta direccionalidad se aprecia la importancia de la cultura en la formación integral del estudiante universitario, algunas normativas legales que sustentan la misma y su la gestión cultural endógena y exógena, presente en la función de extensión universitaria y desarrollada en las diversas Direcciones 
de Cultura de las casas de estudios superiores para la integración comunitaria, con el desarrollo de proyectos y programas permanentes que permiten abordar realidades desde la interrelación de diferentes disciplinas, comprender el mundo actual y aportar soluciones a los problemas sociales.

En sintonía con lo antes descrito, Pernía (2018): indica que "la responsabilidad social universitaria debe promover una perspectiva reflexiva de los gerentes académicos para trascender la labor formativa, desde una mirada prospectiva estratégica en un pensamiento sistémico" (pág. 330); sin muros disciplinarios a fin de crear una gestión con conocimientos congruentes a las necesidades del país en el compromiso de optimizar la calidad de vida.

La premisa anterior fundamenta que la (RSU) está directamente relacionada con la misión universitaria, sus procesos de gestión, docencia, investigación y extensión bajo un compromiso social activo, democrático y participativo orientado a la solución de sus problemas, lo que confirma la relevancia de los desafíos de la responsabilidad social desde la extensión universitaria dirigidos a atender las necesidades de las comunidades y promover el desarrollo sociocultural del país desde su alma mater.

\section{Fundamentación Onto-epistemológica y Metodológica}

\subsection{Paradigma y Enfoque de la Investigación}

Esta investigación doctoral se inscribe en el paradigma interpretativo el cual, Kuhn (1971): plantea que el “...paradigma es un esquema teórico, o una vía de percepción y comprensión del mundo, que un grupo de científicos ha adoptado" (pág. 57). Un género de vida provocado por una forma de interrelaciones socioculturales que incumbe a un periodo determinado en el tiempo, semejante a particularidades del discernimiento de las personas en un espacio histórico que conlleva a lo general. Asimismo, se enmarca con un enfoque cualitativo que según Denzin y Lincoln (2005): “...la investigación cualitativa es interpretativa y naturalista" (pág. 2); significando que un estudio 
cualitativo exige ubicarse en estado natural frente a las situaciones del fenómeno abordado para convertirse en interpretaciones del objeto de estudio.

La Investigación se asienta en el paradigma interpretativo con un enfoque cualitativo, este modelo paradigmático se ocupa del estudio de las expresiones y conductas humanas en los contextos propios de su naturaleza fenomenológica, en una realidad que no se construye, sino que se da por sí misma, reflejada en modos de vida, de actuación, de relaciones e interacciones en todos los campos de saberes de la gerencia de las Direcciones de Cultura, sujetos de estudio y en este caso en el campo de labores del contexto de estudio.

\subsection{Instancia Ontológica}

Esta instancia ontológica, Según Sandín (2003): involucra "...Descripciones detalladas de situaciones, eventos, personas... incorporando la voz de los participantes sus experiencias" (pág. 121); es la manera cómo la investigadora se involucra con los actores sociales sujetos de estudio, indicando cómo será descrita la realidad donde se desarrollan los hechos observados; cuáles fueron esos espacios sociales, en este caso las Universidades objeto de estudio.

Asimismo, en cuanto a la intersubjetividad, complemento esta aseveración, según Gergen (1990), citado por Hernández y Sánchez (2015): se concibe como "aquellas... que permiten la comprensión del conocimiento partiendo del intercambio activo y comunal entre las personas y el contexto sociocultural" (pág. 7); de los hombres conviven en ese mundo, vinculándose unos con otros en sus quehaceres comunes, intervenido por gestos y palabras. Doy por sentado entonces, que el contexto de estudio de esta investigación han sido los espacios de trabajo cotidianos de los informantes clave de las universidades seleccionadas para esta investigación, gestores de las Direcciones de Cultura, lo cual me permitió interactuar con los actores sociales 
a través de las entrevistas en profundidad.

\subsection{Método Fenomenológico-Hermenéutico}

El método utilizado en función de la naturaleza del estudio es el fenomenológico-hermenéutico o interpretativo, el cual según Heidegger (1974): "es donde se articula la hermenéutica al contexto de la temporalidad y la historicidad de la existencia humana" (pág. 67). La hermenéutica alumbra las vivencias. Se devela en la interacción sociocultural donde fluye la universalidad la preeminencia de lo compartido en el entorno. Un entorno donde los actos comunicativos prevalecen.

Por otra parte, Martínez (2006): enfatiza que este método es utilizado cuando es necesario estudiar el fenómeno desde "el marco de referencia del sujeto que las vive y experimenta... una realidad cuya esencia depende del modo en que es vivida y percibida por el sujeto, una realidad interna y personal, única y propia de cada ser humano" (pág. 137). El método fenomenológico hermenéutico según el autor citado se estructura a través de fases donde se despliegan situaciones vivenciales y apreciaciones de las personas para la construcción de la teórica.

Como informantes clave, se seleccionaron los directores de cultura de: Universidad de Carabobo, Universidad Pedagógica Experimental Libertador de Caracas, Universidad "Rafael Belloso Chacín" de Maracaibo, y Universidad Politécnica Territorial "Andrés Eloy Blanco" de Barquisimeto, por ser quienes manejan, desarrollan y toman decisiones en sus respectivas direcciones.

La técnica escogida conseguir la información fue la entrevista a profundidad la cual, en criterio de Rodríguez, Gil y García (1996): "Es la descripción de los significados vividos, existenciales, procurando explicar los significados en los que estamos inmersos en nuestra vida cotidiana" (pág. 40); es una pericia en la que un individuo requiere información de otra persona para conseguir fundamentos sobre el inconveniente explícito, en este caso los 
relatos de los directores de cultura sobre el accionar de la gerencia cultural como factor de cumplimiento y desarrollo de la Responsabilidad Social Universitaria en Venezuela.

Recaudada la información, se procedió a su interpretación, procedimiento indicado por Martínez (2004): “...El proceso completo implica la categorización, la estructuración individual y general, la contrastación y la teorización propiamente dicha" (pág. 21). Respecto a la categorización, se transcribió la información obtenida y se organizaron contenidos por unidades temáticas, que enuncian ideas o conceptos particulares, luego se construyeron las categorías descriptivas basadas los aportes suministrados por los informantes clave, se inició el proceso de lectura para pensar sobre la información de un modo organizado, revelando eventos, experiencias particulares, características, de donde se obtuvieron las categorías y subcategorías emergentes.

\section{Hallazgos}

Los propósitos de este estudio se sustentaron en los conocimientos y vivencias de los informantes clave en el sentido de concebir un constructo teórico, gerencial de las Direcciones de Cultura y la Responsabilidad Social Universitaria desde una cosmovisión integrativa. La Responsabilidad social involucra prácticas gerenciales de Direcciones de Cultura a fin de generar conocimientos a partir de percepciones, significados y datos semánticos aportados por cada informante clave. Desde ese escenario surgen las siguientes categorías de análisis: Gerencia Cultural Universitaria, Responsabilidad Social Universitaria, Transformación Sociocultural Transcompleja y Uso de Aplicaciones Tecnológicas.

La gerencia Cultural Universitaria desarrollada por las Direcciones de Cultura de las (IES) es planificada en diversas modalidades para atender las necesidades sociales del entorno y propiciar el intercambio de saberes, calidad 
de vida y bienestar social colectivo. La cultura es indispensable para integrar conocimientos y promover la creatividad, a través de la extensión cultural, se desarrollan actividades vinculantes con investigación y docencia tendentes a la trasformación sociocultural.

En la Categoría Responsabilidad Social Universitaria se descubre la posibilidad de aumentar el campo de acción de la cultura, desarrollar la sensibilidad artística de producir proyectos de formación trasversal de formación permanente dirigidos a estimular los niveles de compromiso social desde la dimensión ética y propiciar la relación con estudiantes, egresados, empresas y comunidades a fin de canalizar su aporte a través de la universidad.

Respecto a la Categoría: Transformación Sociocultural Transcompleja, en el marco de creación de nuevos escenarios desde las Direcciones de Cultura, para la reflexión, discusión, emerge el momento de reformular los modelos epistemológicos y organizacionales, la investigación, las estructuras universitarias, el momento de transfigurar los viejos esquemas, promover una visión holística y un Liderazgo Transformador que genere aportes Transdisciplinarios, desde la Redimensión de la Relación UniversidadComunidad.

Desde la perspectiva de la Categoría: Aplicaciones Tecnológicas, la gestión cultural se activa se hace presente en el territorio y se extiende en conexión con su pueblo, mediante el uso de nuevos conocimientos con las cuales se ha creado un vínculo permanente de trabajo proporcionando una interrelación dinámica dentro del desarrollo función de extensión cultural universitaria. Las dependencias culturales deben desarrollar una gerencia basada en las nuevas tecnologías para garantizar la eficiencia y efectividad en el desarrollo de sus procesos gerenciales, con comunicaciones permanentes el uso de redes y uso de ofimática para el trabajo cultural.

La Gerencia Cultural en el contexto de la Responsabilidad Social 
Universitaria posee compromiso indelegable e impostergable con la sociedad. La producción intelectual concebida en este estudio de investigación la he denominado: Gerencia de las Direcciones de Cultura y Responsabilidad Social Universitaria desde una Cosmovisión Integrativa, producto contribuciones facilitadas por cada uno de los informantes que participaron en este estudio. Por lo tanto, la intención fue sistematizar este nuevo saber para propiciar espacios de reflexión donde pueda socializarse el conocimiento emergente.

\section{A manera de Conclusiones}

La universidad posee un compromiso con la transformación social, de forma voluntaria y permanente por lo que a través de las Direcciones de Cultura universitarias se planifican, organizan y ejecutan actividades transdisciplinarias dirigidas a las comunidades para brindar respuestas a la sociedad a través de la extensión cultural universitaria conectada de forma horizontal a la docencia y la investigación para apropiarse de la realidad extramuros y decodificar los horizontes que debe transitar la organización universitaria en su rol integrador y transformador.

Se requiere una visión humanista-científica sistémica de la realidad consustanciada con interdisciplina, cultura, equidad, ecología, ciencia natural y social, unir lo científico y lo tradicional, correlacionar la diversidad biológicacultural a fin de fortalecer la integración de saberes y conocimientos desde nuevos espacios.

El principio filosófico que orienta la (RSU), desde las Direcciones de Cultura universitaria es el humanismo afianzado en una visión ética, con sus grupos de interés, en la vinculación con entes públicos y privados, los valores compartidos y la integración comunidad-universidad, donde la responsabilidad social trasciende el compromiso legal para influir en el desempeño interprofesional de los egresados y producir un cambio social coherente a la realidad, desde una visión interdisciplinar y sistémica. 
En los principios axiológicos de la (RSU) presentes en las Direcciones de Cultura universitaria se enmarcan en la ética dentro los valores compartidos, libertad, tolerancia, respeto, paz, honestidad, entre otros, y pueden promover cambios sustanciales en las competencias de los estudiantes y egresados en la búsqueda de una visión holística en los futuros profesionales universitarios.

Los principios praxeológicos de la (RSU) desde el contexto de la gerencia cultural emergen dentro del concepto de un ser humano-cultural que evoluciona, en red comunicacional activa de nuevas tecnologías, en un entorno globalizado y complejo, la gestión social desarrollada en las (IES), se encuentra asociada a la difusión cultural, al autodesarrollo como a las actividades extensionistas en las comunidades internas y externas, organizada por profesores, artistas en el área de música, danza, artes plásticas, literatura, teatro entre otros.

Las Direcciones de Cultura universitaria poseen equipos interdisciplinarios constituidos en diversas áreas artísticas que hacen uso de las aplicaciones tecnológicas para movilizar las comunicaciones entre los equipos culturales, desarrollar actividades de difusión cultural y propiciar en las comunidades la formación para el uso de las nuevas tecnologías.

La gerencia cultural realiza una gestión extensionista en la formación de los estudiantes y las comunidades en la conjunción de conocimiento, saberes y experiencias en el contexto sociocultural, imprescindible en la construcción del aprendizaje multidisciplinario. Se concluye que la educación superior debe proporcionar espacios para promover el intercambio entre conocimientos y saberes desde una postura crítica y creativa en unión con diversas disciplinas para la formación integral, interdisciplinaria dirigida al bienestar físico y mental, fortaleciendo la formación profesional el accionar, científico, cultural, tecnológico, ambiental hacia el beneficio de la colectividad. 


\section{Referencias}

Chirinos Y., \& Pérez C. (2016). La Responsabilidad Social Universitaria: emprendimiento sostenible como impacto de intervención en comunidades vulnerables. Revista Escuela de Administración de Negocios, (81), 91-110, ISSN: 2590-521X. Recuperado de:

https://doi.org/10.21158/01208160.n81.2016.1560

Denzin, N., \& Lincoln, Y. (2005). The Sage Handbook of Qualitative Research. Third Edition. Thousand Oaks: Sage Publications, Inc. Introduction. The Discipline and Practice of Qualitative Research: pp. 113.

González, L. (2015). La gerencia del Talento Humano para un Liderazgo gerencial en la Gestión Universitaria. Revista ASA, 101-108, ISSN: 2343-6115. Recuperado de: https://revistas.uclave.org/index.php/asa/

Goyo, A., Figueredo, C., Méndez, E., Chirinos, E., \& Rivero, E. (2012). La Gerencia de la educación Universitaria, en la Perspectiva de la Transcomplejidad. Orbis. Revista Científica Ciencias Humanas, 8(23), 109-131, ISSN: 1856-1594. Recuperado de:

http://www.redalyc.org/articulo.oa?id=70925416004

Heidegger, M. (1974). El Ser y el Tiempo. México: Federación Canófila mexicana.

Hernández, D. \& Sánchez, F. (2015). Estrategias de Aprendizaje Heurísticas para la Comprensión Social del Conocimiento. EDUCARE, 19(3), 3-27, ISSN: 2244-7296. Recuperado de:

http://revistas.upel.edu.ve/index.php/educare/article/view/2637

Jaspe, A. (2010). La Gestión de la Extensión Universitaria y el Compromiso Social en las Instituciones de Educación Superior. Notas de Investigación, XII(14), 29-49, ISSN: 1315-552. Recuperado de: https://issuu.com/edea.unesr/docs/notasinvestigacion nro 14

Kuhn, T. (1971). La estructura de las revoluciones científicas. México: 
Fondo de Cultura Económica.

Ley de Universidades (1970). Artículo 138. Caracas, Venezuela: Editorial La Torre.

López, M., Zalthen, L., Hernández, L., \& Cervantes, M. (2016). La Responsabilidad Social Universitaria desde la Perspectiva del Alumno. Ra Ximhai, 12(6), 305-314, ISSN: 1665-0441. Recuperado de: http://www.redalyc.org/articulo.oa?id=46148194021

Martínez, M. (2004). Seminario Taller Metodología Cualitativa. Mimeografiado. Departamento de Ciencia y Tecnología del Comportamiento. Venezuela: Universidad Simón Bolívar.

Martínez, M. (2006). Ciencia y Arte en la Metodología Cualitativa. México: Editorial Trillas.

Morín, E. (2002). Los siete saberes necesarios para la educación del futuro. París. Francia: Ediciones UNESCO.

Pérez, T. (2016). Actualización de la Extensión Universitaria desde una perspectiva compleja. Extensión en Red, (7), 47-62. ISSN: 1852-9569. Recuperado de:

http://perio.un/p.edu.ar/ojs/index.php/extensionenred/article/view/3542

Pernía, J. (2018). Visión de la Gestión en la Gerencia de la Responsabilidad Social Universitaria. Revista Scientific, 3(8), 319333. Recuperado de: https://doi.org/10.29394/Scientific.issn.2542$\underline{2987.2018 .3 .8 .17 .319-333}$

Rivero, E. (2011). La Gerencia Universitaria desde una Visión Humanista. UNESR Gerencia 2000, (10), 19-35, ISSN: 1317-0031. Recuperado de: https://issuu.com/edea.unesr/docs/ug2000 n10

Rodríguez G., Gil, J., \& García E. (1996). Metodología de la Investigación cualitativa. Archidona, Málaga, España: Ediciones Aljibe.

Sandín, M. (2003). “Investigación Cualitativa en Educación. Fundamentos y Tradiciones". Madrid, España: Mc Graw and Hill Interamericana de 


\section{España.}

Sarmiento. A. (2007) Propuesta de un modelo de gerencia para la editorial universitaria venezolana. Revista de Artes y Humanidades UNICA, 8(19), 36-63, ISSN: 1317-102X. Recuperado de:

http://www.redalyc. org/articulo.oa?id=170118451003

Tünnerman, C. (2013). Diez respuestas de la educación superior a los desafíos contemporáneos. Universidades, (56), 5-14, ISSN: 00418935. Recuperado de:

http://www.redalyc.org/articulo.oa?id=37331245002 


\section{Sandra Isabel García Arévalo}

e-mail: sandragarciarte@gmail.com

Nacida en Barquisimeto estado Lara, Venezuela.

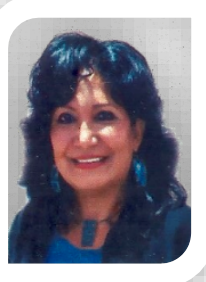

Profesora en Estética de la Universidad Pedagógica

Experimental Libertador (UPEL) y Licenciada en Artes

Plásticas: Mención Museología en la Universidad

Católica Cecilio Acosta. Especialista en Andragogía, Universidad Rafael Urdaneta, Magíster en Gerencia Educativa (UPEL), Doctorado en Gerencia Avanzada en la Universidad Fermín Toro (UFT), se ha desempeñado como Directora de la Escuela de Artes Martin Tovar y Tovar, Profesora Titular por concurso de oposición en la Universidad Centroccidental Lisandro Alvarado, en categoría Agregado, actualmente se desempeña como Coordinadora de Artes Plásticas en la Dirección de Cultura de la (UCLA).

El contenido de este manuscrito se difunde bajo una Licencia de Creative Commons ReconocimientoNoComercial-Compartirlgual 4.0 Internacional 